\title{
ARTICLE
}

Clinical Study

\section{Phase 1 trial of dasatinib combined with afatinib for epidermal growth factor receptor- (EGFR-) mutated lung cancer with acquired tyrosine kinase inhibitor (TKI) resistance}

\author{
Ben C. Creelan ${ }^{1}$, Jhanelle E. Gray ${ }^{1}$, Tawee Tanvetyanon ${ }^{1}$, Alberto A. Chiappori ${ }^{1}$, Takeshi Yoshida ${ }^{2}$, Michael J. Schell ${ }^{3}$,
} Scott J. Antonia ${ }^{1}$ and Eric B. Haura ${ }^{1}$

BACKGROUND: Bypass activation of Src family kinases can confer resistance to EGFR tyrosine kinase inhibitors (TKIs) based on preclinical models. We prospectively assessed the safety and clinical activity of dasatinib and afatinib in combination for patients with resistant EGFR-mutant lung cancer.

METHODS: An open-label, dose-escalation phase 1/2 trial (NCT01999985) with 2-stage expansion was conducted with 25 lung cancer patients. Dose expansion required activating EGFR mutations and progression following prior EGFR TKI.

RESULTS: Patients were $72 \%$ Caucasian and received median of 2 prior lines of therapy. Maximum-tolerated dose was 30 mg afatinib with $100 \mathrm{mg}$ dasatinib. New or increased pleural effusions were observed in $56 \%$ of patients. No radiologic responses were observed, although several EGFR-mutant TKI-resistant patients (26\%) had prolonged stable disease over 6 months. The combination reduced the EGFR mutation and T790M variant allele frequency in cell-free DNA $(p<.05)$. Nonetheless, the threshold for futility was met, based on 6-month progression-free survival. For EGFR TKI-resistant patients, median progression-free survival was 3.7 months (95\% confidence interval $(\mathrm{Cl}), 2.3-5.0)$ and overall survival was 14.7 months (95\% $\mathrm{Cl}, 8.5-20.9)$.

CONCLUSIONS: The combination had a manageable toxicity profile and in vivo T790M modulation, but no objective clinical responses were observed.

British Journal of Cancer (2019) 120:791-796; https://doi.org/10.1038/s41416-019-0428-3

\section{BACKGROUND}

Despite progress in targeting epidermal growth factor receptormutant (EGFRm) non-small cell lung cancer (NSCLC), acquired resistance to EGFR tyrosine kinase inhibitors (TKIs) is inevitable. Common mechanisms include secondary EGFR mutations, such as T790M, bypass receptor tyrosine kinase activation, such as through MET amplification, or tumour reprogramming through epithelial-mesenchymal transition or transformation to small cell lung cancer. Another mechanism is through signalling from Src family kinases (SFKs). Constitutive activation SFKs may contribute to EGFR TKI resistance, in part through ligand-independent activation of c-met. ${ }^{1}$ By combining dasatinib with the irreversible EGFR TKI afatinib, we observed synergistic lethality in EGFRm TKIresistant cell lines and significant tumour reduction in mice bearing EGFR-mutant xenografts harbouring the T790M mutation. $^{2}$ In cell lines, dasatinib overcomes downstream kinase activation associated with T790M TKI resistance, including Src, Akt, and Erk. ${ }^{3}$ High levels of phospho-Src have been identified in tumours after EGFR TKI resistance has been reached. ${ }^{4}$ Loss of PTEN may also contribute to TKI resistance via downstream Src/Akt pathway activation. ${ }^{5,6}$ Overexpression of the Yes-associated protein $Y A P$ is associated with resistance to first-generation EGFR
TKIs. ${ }^{7}$ Overexpression of the EGF-CFC (epidermal growth factorCripto-1/FRL-1/Cryptic) protein family member CRIPTO-1 has been identified to directly enable EGFR resistance through SRC activation $^{8}$ and has been associated with primary TKI resistance among EGFRm patients. ${ }^{9}$ More recent studies using transposon mutagenesis assays identified the SFK member YES1 as a mediator of resistance to all three generations of EGFR TKIs and conferred sensitivity to dasatinib. ${ }^{10,11}$ SFKs appear to sustain AKT and mitogen-activated protein kinase (MAPK) pathway signalling during osimertinib treatment, and combined dasatinib and osimertinib causes inhibition of cancer growth, apoptosis, and delay of acquired resistance in EGFRm animal studies. ${ }^{12}$ Therefore, disruption of the SFK pathway may still remain a viable method of overcoming TKI resistance, and further study of novel approaches is warranted.

Dasatinib is a potent inhibitor of several tyrosine kinase families, including the SFKs. In two phase 2 trials, the combination of dasatinib and erlotinib mediated tumour reductions in two EGFRm patients with acquired resistance, but overall response rates remained low. ${ }^{4,13,14}$ Based on our preclinical studies demonstrating improved in vivo efficacy of dasatinib and afatinib in T790M models of acquired resistance to first-generation EGFR TKI, we

\footnotetext{
${ }^{1}$ Thoracic Oncology, H. Lee Moffitt Cancer Center and Research Institute, 12902 Magnolia Dr., Tampa, FL 33612, USA; ${ }^{2}$ Department of Medical Oncology, Kindai University Faculty of Medicine, 377-2, Ono-Higashi, Osakasayama, Osaka 589-8511, Japan and ${ }^{3}$ Department of Biostatistics and Bioinformatics, H. Lee Moffitt Cancer Center and Research Institute, 12902 Magnolia Dr., Tampa, FL 33612, USA

Correspondence: Ben C. Creelan (ben.creelan@moffitt.org)
}

Received: 6 November 2018 Revised: 19 February 2019 Accepted: 27 February 2019

Published online: 18 March 2019 


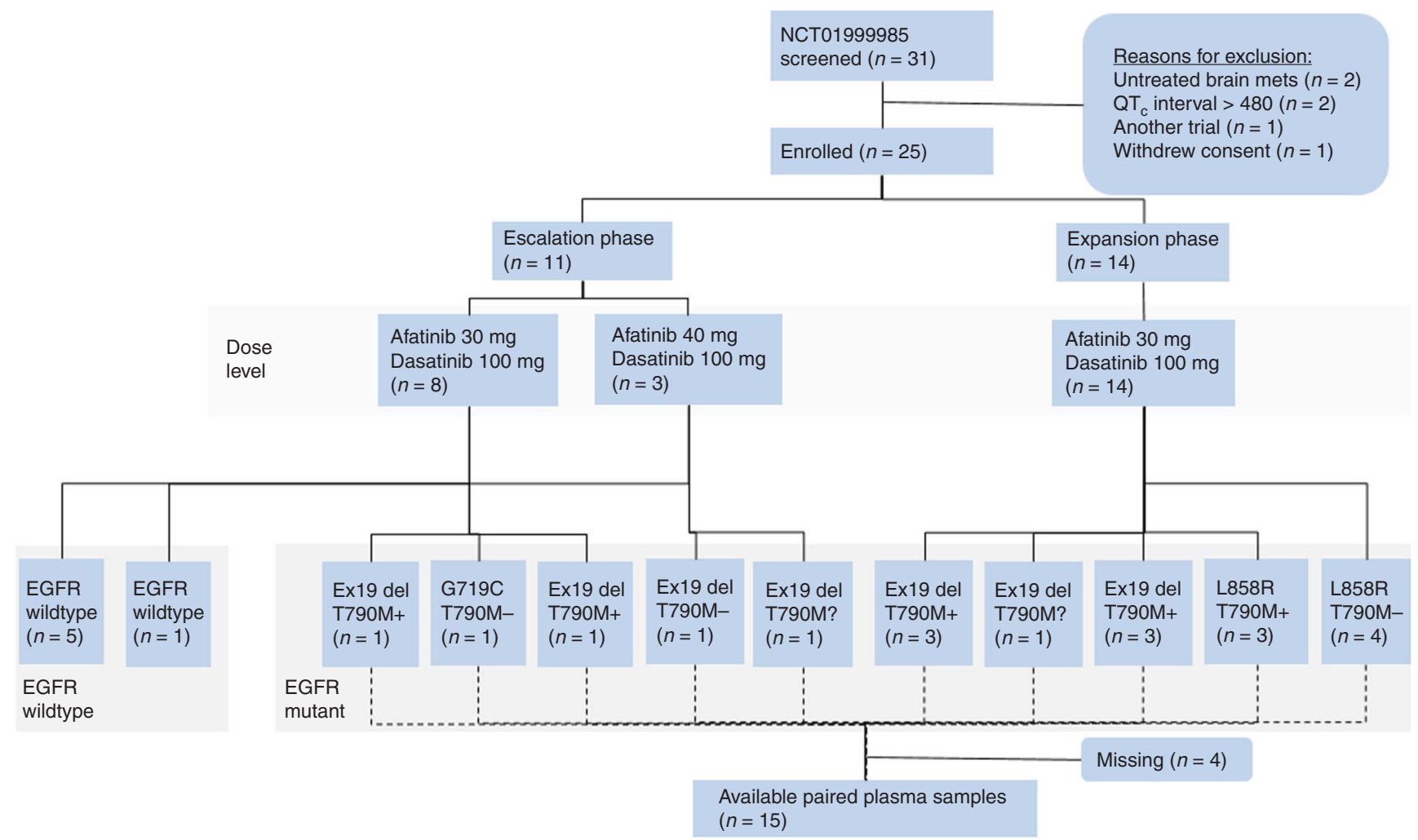

Fig. 1 Diagram showing patient treatment assignment, baseline epidermal growth factor receptor (EGFR) status, and sample availability. All patients had first-line epidermal growth factor receptor tyrosine kinase inhibitor resistance. EGFR mutation status assessed by plasma or tissue test: T790M+ detected, T790M - not detected, T790M? unknown

hypothesised that this combination would lead to durable disease control in EGFRm NSCLC patients with acquired TKI resistance. Therefore, we conducted a phase $1 / 2$ trial with the primary objective of characterising the safety and clinical activity of dasatinib with afatinib in this population. We also hypothesised that assessment of cell-free DNA for EGFR mutations could serve as an additional readout of drug efficacy.

\section{MATERIALS AND METHODS}

This was an open-label, single-centre, phase 1 study with a modified $3+3$ dose-escalation design, followed by an expansion cohort with a 2-stage design (NCT01999985). The trial was approved by Liberty Institutional Review Board Inc., assurance number IRB00003411. For dose escalation, patients were required to have stage 4 NSCLC with progression after $\geq 1$ standard therapy. For dose expansion, patients were required to have an activating EGFR exon 19 or 21 mutation, with disease progression after $\geq 1$ TKI (gefitinib, afatinib, or erlotinib). On the basis of the concentrations observed to modulate Src in vivo, starting doses of $100 \mathrm{mg}$ dasatinib and $30 \mathrm{mg}$ afatinib once daily were chosen. T790M positive was defined as the presence of detectable allele in either tumour or plasma. Patient with pericardial or pleural effusion of grade 2 or higher were excluded. Patients were required to have RECIST evaluable disease. Enlargement of pre-existing pleural effusions by itself was interpreted cautiously, and not counted as a non-target progression without convincing evidence.

The sample size of the escalation cohort was powered to conclude the dose-limiting toxicity (DLT) rate was $\leq 33 \%$ if no DLTs were observed in 8 patients treated at the dose level, with $85 \%$ power and a 1-sided a of 0.10 . For the expansion cohort, the null hypothesis was that $26 \%$ of patients would be progression free at
6 months, and the alternative hypothesis was that true progression-free survival would be 6.6 months, e.g., a progression-free rate of $52 \%$. Using one-sided binomial test with actual $\mathrm{a}=0.046$, this design had $66 \%$ power with $n=14$. For an expanded description of the methods involved in the study, please see Supplementary Material: Methods.

\section{RESULTS}

Patient characteristics

Of the 31 patients screened, 25 were eligible and treated (Fig. 1). Patients had received a median of 2 (range, 1-5) prior systemic therapies for stage 4 NSCLC and had progressive measurable disease (Supplemental Table S1). Patients with an activating EGFR mutation had previously progressed on a first- or secondgeneration EGFR TKI. No patients had evidence of small cell transformation, and none had received prior third-generation TKIs, such as osimertinib.

Safety and tolerability

Dose escalation proceeded through 2 dose levels of up to $40 \mathrm{mg}$ afatinib with $100 \mathrm{mg}$ dasatinib. Although no protocol-defined DLT was observed, we decided to deescalate after 3 patients were enrolled at the $40 \mathrm{mg}$ dose level, due to the persistence of grade 2 diarrhoea despite optimal medical management in 2 patients. These two events were therefore recorded as DLTs for monitoring purposes.

Patients received a mean of 3.2 months of continuous oral drug exposure. Adverse events (AEs) for both dose levels in all patients are summarised together (Fig. 2). The most common drug-related AEs were diarrhoea (72\%) and rash (64\%) (Supplemental Table S2). The most common serious AEs, regardless of causality, were pneumonia (24\%), diarrhoea (12\%), and pleural effusion (12\%). 


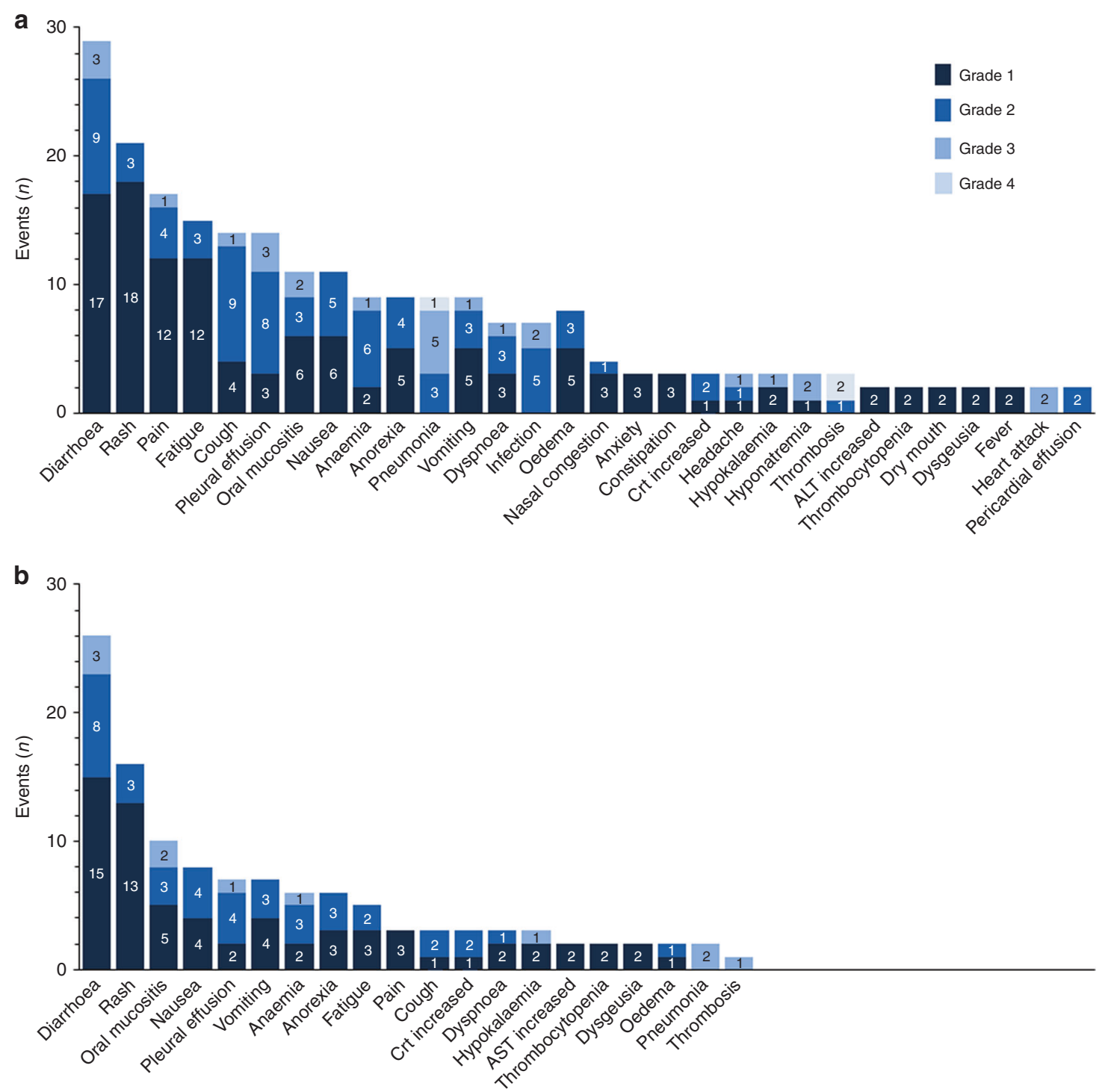

Fig. 2 Graphs show all adverse events occurring more than once, and any event grade $\geq 2$ in severity. Numbers of adverse events for each category are shown in the bars. a Total adverse events $(n=229$ are shown). b Treatment-related adverse events $(n=117$ are shown). ALT alanine transferase, Crt creatinine

Although drug-related AEs were generally manageable, $24 \%$ of patients eventually required dose reductions or interruptions for the management of AEs.

New or increased pleural effusions occurred in $72 \%$ of patients on reimaging computed tomography (CT) scans (Supplemental Table S3). In many instances, it was difficult to ascertain whether effusions were attributable to dasatinib or lung cancer, since enlargement of pre-existing malignant effusions could be attributable to either cause. Symptomatic pleural effusions were managed with thoracentesis, corticosteroid tapers, or dose reductions of dasatinib to $50 \mathrm{mg}$ daily, if recrudescence occurred.

No QTc prolongations on serial electrocardiograms were detected during the trial (Supplemental Fig. 1A). No decrease in mean left ventricular ejection fraction below normal levels $(<50 \%)$ was detected (Supplemental Fig. 1B).

Pharmacodynamics

For patients with activating EGFRm with acquired TKI resistance, an overall decrease in EGFRm-variant allele frequency was observed during the course of treatment (Fig. 3a). The interpretation was limited by a high degree of deviation in variant allele frequencies between patients. Likewise, a decrease in T790M allele frequency was observed among patients with baseline detectable T790M (Fig. 3b). Samples for subsequent time points were not collected.

Treatment outcomes and overall survival No radiologic responses were observed, although several EGFRm TKI-resistant patients had stable disease as best response (Fig. 4). One patient with acquired T790M had stable disease lasting more than 12 months. The trial met the interim stopping threshold for futility based upon 6-month progression-free survival. For all EGFR TKI-resistant patients, median progression-free survival was 3.7 months (95\% confidence interval $(\mathrm{Cl}), 2.3-5.0)$. Median overall survival was 14.7 months $(95 \% \mathrm{Cl}, 8.5-20.9)$ at a median follow-up of 25 months. EGFRm patients lacking detectable T790M at entry had worse overall survival than those with detectable T790M at entry, with a hazard ratio of $4.0(95 \% \mathrm{Cl}, 1.3-13.1), p=.02$. This may be partly attributable to subsequent osimertinib as the next 
794
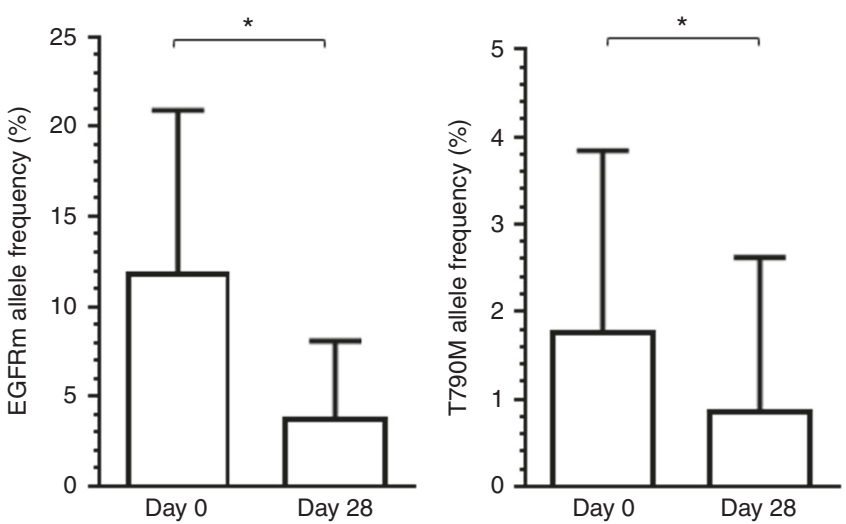

Fig. 3 Decrease in mutant allele fraction from plasma cell-free DNA during afatinib-dasatinib treatment. Patients with activating epidermal growth factor receptor mutations had paired samples assessed by digital droplet polymerase chain reaction. Fifteen patients were evaluable. Bars represent mean values with $95 \%$ confidence intervals. ${ }^{*} P<.05$ by Wilcoxon matched-pairs signedrank test

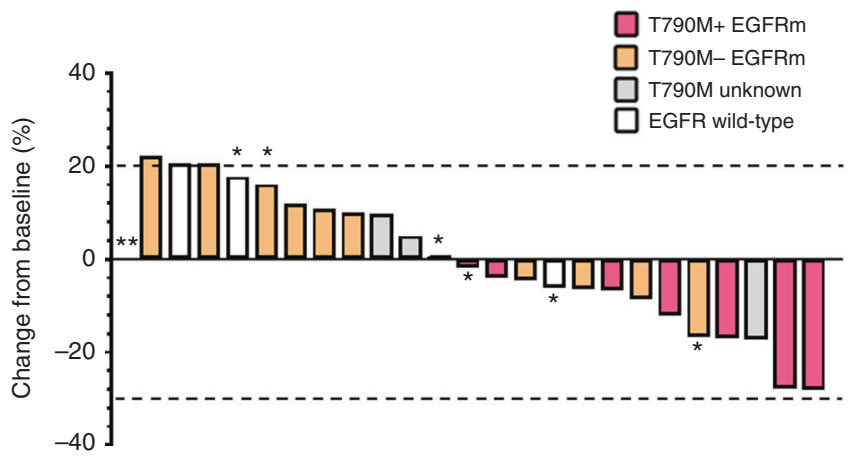

Fig. 4 Waterfall plot shows best percentage changes in sums of target lesions compared to baseline. *Progression by non-target or new lesion. **Target non-evaluable at progression line of therapy for patients who were T790M positive at study entry, as shown in Fig. 5. All deaths were attributable to progressive cancer. No ostensible clinical benefit was detected in the EGFR wild-type population, with a median progression-free survival of 1.8 months $(95 \% \mathrm{Cl}, 0.7-3.0)$ and median overall survival of 5.1 months $(95 \% \mathrm{Cl}, 2.4-7.8)$.

\section{DISCUSSION}

In this phase 1 trial, it was feasible to combine dasatinib with afatinib in advanced NSCLC at biologically active doses. Nonetheless, pleural effusion remained a prominent adverse effect, with most patients who had been effusion free at study entry exhibiting pleural effusions on their reimaging $C T$ scans. The inclusion of patient with mild pleural effusions may have confounded the interpretation of this adverse effect. Nevertheless, our goal was to enrol representative patients, since malignant pleural effusions occur in almost $40 \%$ of metastatic NSCLC. ${ }^{15}$ Moreover, the clinical activity of the combination was limited and did not justify further investigation, especially since third-generation EGFR TKls such as osimertinib have become available and demonstrate significant responses in a similar patient population.

This combination was observed to modestly decrease the fraction of plasma-mutant EGFR and T790M alleles at week 4. In contrast, complete clearance of plasma T790M occurred at week 6 among most patients on the AURA osimertinib trial and was associated with durable response. ${ }^{16}$ Although dasatinib has modest in vitro binding affinity to mutant EGFR kinases, T790M appears to cause steric hindrance with the chloro-methyl-phenyl ring of dasatinib. ${ }^{17}$ Nonetheless, dasatinib is hypothesised to suppress bypass tracks through SRC/AKT, rather than direct inhibition of the T790M EGFR kinase.

Despite promising preclinical activity, dasatinib is characterised by a short terminal half-life in plasma, which may constrain its inhibitory activity of SFKs in human tissue. ${ }^{18}$ To this end, the reported tolerability and efficacy of dasatinib as a single agent across multiple solid tumours has been unsatisfactory. Two phase $1 / 2$ NSCLC trials of single-agent dasatinib at higher doses were closed due to a high frequency of AEs and a paucity of

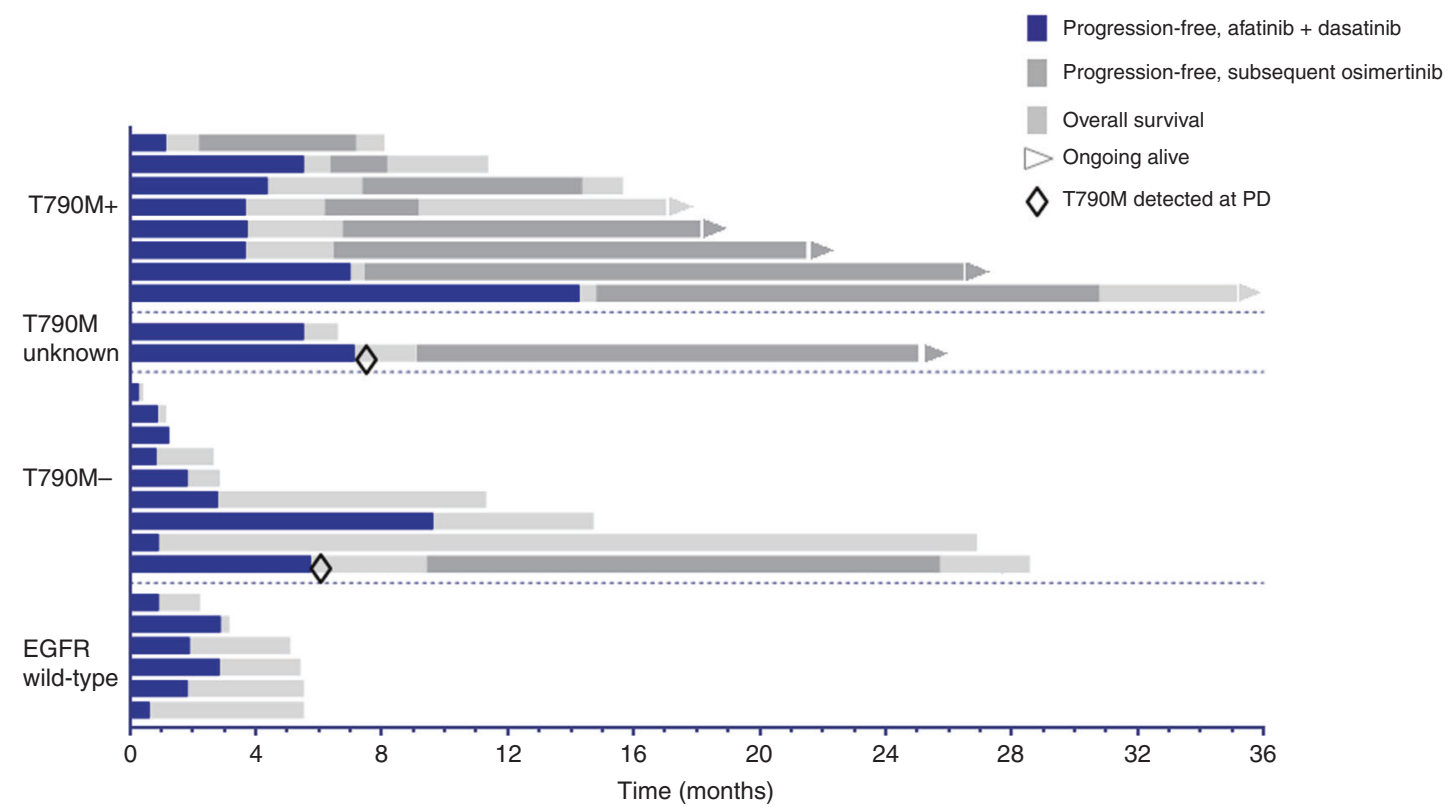

Fig. 5 Swimmers plot shows individual progression-free and overall survival of afatinib-dasatinib patients by baseline epidermal growth factor receptor mutation status 
Phase 1 trial of dasatinib combined with afatinib for epidermal growth... BC Creelan et al.

durable responses. ${ }^{19,20}$ Likewise, additional trials of dasatinib with erlotinib reported low or absent responses in patients with acquired EGFR TKI resistance. ${ }^{13,14}$ Finally, a phase 2 trial of dasatinib for inactivating BRAF mutations was closed due to a lack of efficacy amongst the study population. ${ }^{21}$ Therefore, a trial testing osimertinib with dasatinib in EGFRm NSCLC based upon baseline plasma or tumour CRIPTO-1 expression is ongoing. ${ }^{22}$ Additionally, YAP amplification has been proposed to confer resistance in up to $4 \%$ of EGFR TKI-resistant patients, which may implicate SFK as important in mediating resistance. ${ }^{7}$ Therefore, there may yet be a further role for SFK inhibitors in these narrowly defined molecular subgroups. In our study, tumour biopsies were not performed, and thus we could not detect phospho-Src, YES amplification, or Cripto-1 alterations within the tumour. Future studies of SFK in combination with EGFR targeting agents should focus on subsets of patients with likely mechanisms of resistance driven by SFK.

\section{ACKNOWLEDGEMENTS}

Trial was designed at the AACR/ASCO 2011 Methods in Cancer Research workshop, including assistance from mentors Patricia LoRusso, Gary Clark, and Miguel Villalona. Angela Reagan and Sonya J. Smyk provided editorial assistance. Angela Reagan and Sonya J. Smyk was not compensated beyond her regular salary. This study was partially funded by the Moffitt Cancer Center National Cancer Institute support grant, P30-CA076292. Afatinib drug supply and funding for the conduct of the clinical trial was provided by Boehringer Ingelheim $\mathrm{GmbH}$. Dasatinib drug supply was provided by E.R. Squibb \& Sons, L.L.C. Support for the performance of digital droplet PCR was provided by Biodesix, Inc. The support sources had no role in the design, analysis or interpretation of the results in this study. BIPI was given the opportunity to review the manuscript for medical and scientific accuracy as it relates to BIPI substances, as well as intellectual property considerations.

\section{AUTHOR CONTRIBUTIONS}

The authors meet criteria for authorship as recommended by the International Committee of Medical Journal Editors (ICMJE). B.C.C. was the principal investigator who helped to design, conducted the trial, analysis of results, and wrote the primary manuscript. J.E.G. helped by reviewing trial design, contributing patients, and review of the manuscript. T.T. helped by contributing patients and manuscript review. A.A.C. helped by reviewing trial design, contributing patients, and review of the manuscript. T.Y. helped by writing the protocol rationale, and review of the manuscript. M.J.S. provided statistical justification for the study design, analysis, and review of the manuscript. S.J.A. helped by contributing patients and review of the manuscript. E.B. B. provided the study rationale, supervised the study design, contributed patients, and contributed to the analysis and manuscript review.

\section{ADDITIONAL INFORMATION}

Supplementary information is available for this paper at https://doi.org/10.1038/ s41416-019-0428-3.

Competing interests: B.C.C. discloses the following: Speaker's Bureau: HoffmanLaRoche AG, E.R. Squibb \& Sons LLC, AstraZeneca LLC, Takeda Pharmaceutical Company Ltd, Foundation Medicine Inc. Advisory Board: E.R. Squibb \& Sons LLC, AbbVie Inc., GlaxoSmithKline plc, Celgene. Contracted/Support Research Grant: Prometheus Inc., lovance Biotherapeutics Inc., Boehringer Ingelheim GmbH. J.E.G. discloses research support from Boehringer Ingelheim $\mathrm{GmbH}$ and Bristol-Myers Squibb. S.J.A. has research funding from Boehringer Ingelheim. E.B.H. serves on the advisory board of Janssen Pharmaceuticals and receives research support from Forma Therapeutics and Incyte Pharmaceuticals. The other authors declare no competing interests.

Ethics approval and consent to participate: The study was institutional review board approved, Federal wide assurance number IRB00003411, Liberty Institutional Review Board, Inc. All patients provided written informed consent. The study was performed in accordance with the Declaration of Helsinki.

Data availability: Raw data and materials generated during the study are available upon request to the author study team, thoraciccro@moffitt.org.
Note: This work is published under the standard license to publish agreement. After 12 months the work will become freely available and the license terms will switch to a Creative Commons Attribution 4.0 International (CC BY 4.0).

Publisher's note: Springer Nature remains neutral with regard to jurisdictional claims in published maps and institutional affiliations.

\section{REFERENCES}

1. Stabile, L. P., He, G., Lui, V. W. Y., Thomas, S. M., Henry, C. \& Gubish, C. T. et al. c-Src activation mediates erlotinib resistance in head and neck cancer by stimulating cMet. Clin. Cancer Res. 19, 380-392 (2013).

2. Yoshida, T., Zhang, G., Smith, M. A., Lopez, A. S., Bai, Y. \& Li, J. et al. Tyrosine phosphoproteomics identifies both codrivers and cotargeting strategies for T790M-related EGFR-TKI resistance in non-small cell lung cancer. Clin. Cancer Res. 20, 4059-4074 (2014).

3. Watanabe, S., Yoshida, T., Kawakami, H., Takegawa, N., Tanizaki, J. \& Hayashi, H. et al. T790M-selective EGFR-TKI combined with dasatinib as an optimal strategy for overcoming EGFR-TKI resistance in T790M-positive non-small cell lung cancer. Mol. Cancer Ther. 16, 2563-2571 (2017).

4. Haura, E. B., Tanvetyanon, T., Chiappori, A., Williams, C., Simon, G. \& Antonia, S. et al. Phase I/II study of the Src inhibitor dasatinib in combination with erlotinib in advanced non-small-cell lung cancer. J. Clin. Oncol. 28, 1387 (2010).

5. Sos, M. L., Koker, M., Weir, B. A., Heynck, S., Rabinovsky, R. \& Zander, T. et al. PTEN loss contributes to erlotinib resistance in EGFR-mutant lung cancer by activation of Akt and EGFR. Cancer Res. 69, 3256-3261 (2009).

6. Kanda, R., Kawahara, A., Watari, K., Murakami, Y., Sonoda, K. \& Maeda, M. et al. Erlotinib resistance in lung cancer cells mediated by integrin $\beta 1 / \mathrm{Src} /$ Akt-driven bypass signaling. Cancer Res. 73, 6243-6253 (2013).

7. Lee, T.-F., Tseng, Y.-C., Nguyen, P. A., Li, Y.-C., Ho, C.-C. \& Wu, C.-W. Enhanced YAP expression leads to EGFR TKI resistance in lung adenocarcinomas. Sci. Rep. 8, 271 (2018).

8. Park, K.-S., Raffeld, M., Moon, Y. W., Xi, L., Bianco, C. \& Pham, T. et al. CRIPTO1 expression in EGFR-mutant NSCLC elicits intrinsic EGFR-inhibitor resistance. $J$. Clin. Investig. 124, 3003-3015 (2014).

9. Zhang, H., Zhang, B., Gao, L., Zhang, L., Zhu, K. \& Cheng, R. et al. Clinical significance of cripto-1 expression in lung adenocarcinoma. Oncotarget 8, 79087 (2017).

10. Fan, P.-D., Narzisi, G., Jayaprakash, A., Venturini, E., Robine, N. \& Smibert, P. et al. YES1 amplification as a mechanism of acquired resistance (AR) to EGFR tyrosine kinase inhibitors (TKIs) identified by a transposon mutagenesis screen and clinical genomic testing. Am. Soc. Clin. Oncol. 35(15_Suppl.), 9043 (2017).

11. Fan, P.-D., Narzisi, G., Jayaprakash, A. D., Venturini, E., Robine, N. \& Smibert, P. et al. YES1 amplification is a mechanism of acquired resistance to EGFR inhibitors identified by transposon mutagenesis and clinical genomics. Proc. Natl. Acad. Sci. USA 115, E6030-E6038 (2018).

12. Ichihara, E., Westover, D., Meador, C. B., Yan, Y., Bauer, J. A. \& Lu, P. et al. SFK/FAK signaling attenuates osimertinib efficacy in both drug-sensitive and drug-resistant models of EGFR-mutant lung cancer. Cancer Res. 77, 2990-3000 (2017).

13. Gold, K. A., Lee, J. J., Harun, N., Tang, X., Price, J. \& Kawedia, J. D. et al. A phase I/II study combining erlotinib and dasatinib for non-small cell lung cancer. Oncologist 19, 1040 (2014).

14. Johnson, M. L., Riely, G. J., Rizvi, N. A., Azzoli, C. G., Kris, M. G. \& Sima, C. S. et al. Phase II trial of dasatinib for patients with acquired resistance to treatment with the epidermal growth factor receptor tyrosine kinase inhibitors erlotinib or gefitinib. J. Thorac. Oncol. 6, 1128-1131 (2011).

15. Porcel, J. M., Gasol, A., Bielsa, S., Civit, C., Light, R. W. \& Salud, A. Clinical features and survival of lung cancer patients with pleural effusions. Respirology 20, 654-659 (2015).

16. Thress, K. S., Markovets, A., Barrett, J. C., Chmielecki, J., Goldberg, S. B. \& Shepherd, F. A. et al. Complete clearance of plasma EGFR mutations as a predictor of outcome on osimertinib in the AURA trial. Am. Soc. Clin. Oncol. 35(15_Suppl.), 9018 (2017).

17. Sos, M. L., Michel, K., Zander, T., Weiss, J., Frommolt, P. \& Peifer, M. et al. Predicting drug susceptibility of non-small cell lung cancers based on genetic lesions. J. Clin. Investig. 119, 1727-1740 (2009).

18. Christopher, L. J., Cui, D., Wu, C., Luo, R., Manning, J. A. \& Bonacorsi, S. J. et al. Metabolism and disposition of dasatinib after oral administration to humans. Drug Metab. Dispos. 36, 1357-1364 (2008).

19. Brunner, A. M., Costa, D. B., Heist, R. S., Garcia, E., Lindeman, N. I. \& Sholl, L. M. et al. Treatment-related toxicities in a phase II trial of dasatinib in patients with squamous cell carcinoma of the lung. J. Thorac. Oncol. 8, 1434-1437 (2013). 
Phase 1 trial of dasatinib combined with afatinib for epidermal growth...

BC Creelan et al.

796

20. Johnson, F. M., Bekele, B. N., Feng, L., Wistuba, I., Tang, X. M. \& Tran, H. T. et al. Phase II study of dasatinib in patients with advanced non-small-cell lung cancer. J. Clin. Oncol. 28, 4609 (2010).

21. Trial of dasatinib in patients with advanced cancers harboring DDR2 mutation or inactivating B-RAF mutation (ed. Medicine UNLo). ClinicalTrialsgov Identifier:
NCT01514864. ClinicalTrials.gov (Bristol-Myers Squibb, 2015) https://clinicaltrials. gov/ct2/show/NCT01514864.

22. Kim, C., Liu, S., Subramaniam, D. \& Giaccone, G. P1.01-47 Phase I/II trial of dasatinib and osimertinib in patients with advanced EGFR-mutant non-small cell lung cancer (NSCLC). J. Thorac. Oncol. 13, S479 (2018). 